\title{
Correction to: Efficacy of electro- acupuncture and manual acupuncture versus sham acupuncture for knee osteoarthritis: study protocol for a randomised controlled trial
}

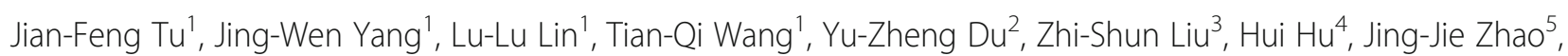
Xiao-Gang Yu ${ }^{6}$, Chun-Sheng Jia ${ }^{7}$, Jun Wang ${ }^{8}$, Tong Wang ${ }^{9}$, Ya-Quan Hou', Xuan Zou ${ }^{1}$, Yu Wang ${ }^{10}$, Jia-Kai Shao ${ }^{10}$, Li-Qiong Wang ${ }^{1}$, Zhang-Sheng $Y u^{11}$ and Cun-Zhi Liü ${ }^{1 *}$

Correction to: Trials (2019) 20:79

https://doi.org/10.1186/s13063-018-3138-x

After publication of the original article [1], the authors have notified us that the Trial registration number NCT03274713 should be replaced by NCT03366363 in the Abstract section of the paper.

\footnotetext{
Author details

${ }^{1}$ School of Acupuncture-Moxibustion and Tuina, Beijing University of Chinese Medicine, Fengtai District, Beijing, China. ${ }^{2}$ Department of Acupuncture and Moxibustion, First Teaching Hospital of Tianjin University of Traditional Chinese Medicine, Xiqing District, Tianjin, China. ${ }^{3}$ Department of Acupuncture and Moxibustion, Guang'an Men Hospital, China Academy of Chinese Medical Sciences, Xicheng District, Beijing, China. ${ }^{4}$ Department of Acupuncture and Moxibustion, Dongfang Hospital, Beijing University of Chinese Medicine, Fengtai District, Beijing, China. ${ }^{5}$ Department of Traditional Chinese Medicine, Beijing Friendship Hospital, Capital Medical University, Xicheng District, Beijing, China. ${ }^{6}$ Department of Acupuncture and Moxibustion, Beijing Hospital of Traditional Chinese and Western Medicine, Haidian District, Beijing, China. ${ }^{7}$ Hebei University of Chinese Medicine, Shijiazhuang, Heibei, China. ${ }^{8}$ Department of Acupuncture and Moxibustion, Dongzhimen Hospital, Beijing University of Chinese Medicine, Dongcheng District, Beijing, China. ${ }^{9}$ Department of orthopedics, Institute of Acupuncture and Moxibustion, China Academy Of Chinese Medicine Sciences, Dongcheng District, Beijing, China. ${ }^{10}$ Department of Acupuncture and Moxibustion, Beijing Hospital of Traditional Chinese Medicine Affiliated to Capital Medical University, Dongcheng District, Beijing, China. ${ }^{11}$ Department of Bioinformatics and Biostatistics, SJTU-Yale Joint Center for Biostatistics, Shanghai Jiao Tong University, Minhang District, Shanghai, China.
}

Received: 2 April 2019 Accepted: 2 April 2019

Published online: 10 April 2019

\section{Reference}

1. Tu JF, et al. Efficacy of electro-acupuncture and manual acupuncture versus sham acupuncture for knee osteoarthritis: study protocol for a randomised controlled trial. Trials. 2019;20:79. https://doi.org/10.1186/s13063-018-3138-x.

* Correspondence: Icz623780@126.com

${ }^{1}$ School of Acupuncture-Moxibustion and Tuina, Beijing University of Chinese Medicine, Fengtai District, Beijing, China

Full list of author information is available at the end of the article

(c) The Author(s). 2019 Open Access This article is distributed under the terms of the Creative Commons Attribution 4.0 International License (http://creativecommons.org/licenses/by/4.0/) which permits unrestricted use, distribution, and reproduction in any medium, provided you give appropriate credit to the original author(s) and the source, provide a link to the Creative Commons license, and indicate if changes were made. The Creative Commons Public Domain Dedication waiver (http://creativecommons.org/publicdomain/zero/1.0/) applies to the data made available in this article, unless otherwise stated. 